\title{
Modifying Cerebrospinal Fluid Constants for the Tuberculousis Etiology of a Meningitis Case
}

\author{
IOANA FLORINA MIHAI ${ }^{1}$, ALEXANDRA LARGU1,2,*, V. DOROBAT ${ }^{3}$, CARMEN MANCIUC ${ }^{1,2}$ \\ ${ }^{1}$ Sfanta Parascheva Infectious Diseases Clinical Hospital lasi, 2 Octav Botez Str., 700116, lasi, Romania \\ ${ }^{2}$ Grigore T. Popa University of Medicine and Pharmacy lasi, 16 Universitatii Str., 700115, Iasi, Romania \\ 3University Emergency Hospital Bucuresti, 169 Splaiul Independentei, 050098, Bucharest, Romania
}

\begin{abstract}
Tuberculous meningitis is the most lethal form of Mycobacterium tuberculosis infection, with a high rate of neurological complications and sequelae. We present the case of a 6 years old child diagnosed with tuberculous meningitis based on epidemiological, clinical and biological data and who has rapidly developed neurological complications with persistent fever and headache for a long time. Under tuberculostatic, depletion and corticosteroid treatment, the evolution of the disease was slowly favorable.
\end{abstract}

Keywords: meningitis, tuberculousis, tuberculostatic therapy, focal neurological disorders

Tuberculosis (TB) is caused by a bacterium called Mycobacterium tuberculosis. In the last decade, it is the world's 9th leading cause of death and the most important disease caused by a single infectious agent (1).

According to the World Health Organization (WHO) report from 2016, over 6 million new cases of active TB have been reported worldwide (2). According to The European Centre for Disease Prevention and Control (ECDC) reports, in the case of TB in children, in 2015, 2,444 cases aged less than 15 years were reported, representing $4.1 \%$ of all TB cases, respectively 3 cases per 100,000 children.

In Romania, tuberculosis is one of the priority issues of public health, with 13,617 new cases registered in 2016, of which 591 in children (1).

Although tuberculosis primarily affects the lungs, it can affect virtually any organ, including the central nervous system (CNS), and can cause tuberculous meningitis (3). The last one is estimated to account for about $1 \%$ of all tuberculosis cases in developed countries (2).

The natural history and clinical manifestations of tuberculosis are different in children and adults, with a higher incidence of disseminated forms and a higher risk for the progression of severe forms reported in children (4). To these, tuberculous meningitis can be devastating, leading to death or permanent disability, but the disease can be prevented and treated if it is diagnosed on time. Once tuberculosis has spread to the brain, it can lead to permanent disabilities such as cerebral palsy, epilepsy, severe behavioral problems and blindness (5).

Tuberculous meningitis (TBM) should be suspected in all children who have signs and symptoms of meningitis (fever, irritability, neck stiffness, lethargy, headache, nausea) or neurological symptoms (seizures, focal neurologic disorders) in case of recent contact with a person with infectious tuberculosis or other risk factors for the development of tuberculosis (6). Children with TBM often have a lower headache intensity, but are more likely to experience signs of meningitis, gastrointestinal symptoms and mental health alterations than adults. Also, seizures are more common in children than in adults $(4,7)$.

Diagnosis of TBM may be difficult and can only be based on clinical findings and microscopic examination of cerebrospinal fluid (CSF) without definitive microbiological confirmation. Certain clinical features, such as longer onset of symptoms, basically insidious onset (more than six days), moderate CSF pleocytosis, predominant lymphocytes and the presence of focal deficiencies increase the probability of TBM (8). The positive diagnosis is the identification of the pathogen in the cerebrospinal fluid by lumbar puncture.

The introduction of treatment with antituberculous, chemotherapeutic agents such as isoniazid, rifampicin and streptomycin, subsequently pyrazinamide and ethambutol to which cortisone has been judiciously associated, improved prognosis. How ever, a more important role than the choice of treatment regimen is to initiate treatment as close as possible to the onset of the disease (9).

BCG vaccination since childhood, is considered to be one of the measures for the prevention of the disease. Two meta-analyzes published in the early 1990s confirmed the efficacy of BCG vaccine against extrapulmonary tuberculosis (mainly meningitis), with efficacy ranging from $64 \%$ to $86 \%$ depending on the study design (10).

Tuberculous control programs initiated by the World Health Organization (WHO) were often overcome with the prevalence of human immunodeficiency virus (HIV). For this purpose, WHO experts have proposed a new strategy, short-term treatment with lasting supervision to stop the current outbreak (10).

\section{Experimental part}

We will detail the case of a male patient C.R., 6 years old, with no significant pathological personal history, presenting in our clinic by transfer from the Pediatric Hospital. Symptomatology reported by the patient and the patient's family (febrility, asthenia, apathy, sleepiness) raises the suspicion of a meningitis, diagnosis confirmed byimaging and laboratory exploration. This study analyzes retrospectively data on symptomatology, paraclinical data, treatment, disease progression, possible complications of acute illness, as well as subsequent re-evaluations performed through hospital ambulatory.

\section{Results and discussions}

A 6-year-old male patient is presented to our clinic with the following symptoms: general influenced state, marked physical asthenia, fever (38.9 grades C), intense headache, nausea, vomiting, inappetence.

The disease started about 4 days prior to admission through a painful abdominal syndrome, fever, nausea and vomiting. First, he presents to the pediatric emergency service, where he is evaluated for surgery. The consultation

\footnotetext{
*email: largualexandra@yahoo.com
} 
excludes a specialized emergency or a need for operation, and abdominal ultrasound does not detect abnormalities. The next day, the patient installs cephalalgia accompanied by photophobia, and when performing the test for meningeal signs, he sketches off the back of his neck. Because the symptoms progressively worsen, the doctor in call in the Infectious Diseases Hospital is contacted, deciding the transfer in the clinic with suspicion of meningitis.

At admission, the clinical examination highlights an altered general condition, restlessness, fever $\left(38^{\circ} \mathrm{C}\right)$, paleness, dehydration, multiple diffuse vulgar warts on the body, normal pulmonary and cardiac functions, depressed abdomen with diffuse sensitivity to deep palpation, physiological intestinal transit and urination, signs of meningeal contracture present (nuchal rigidity, Kernig I). Following detailed anamnesis, we find that the patient is from an area with high incidence of tuberculosis.

A lumbar puncture is urgently performed, showing clear cerebrospinal fluid, with 357 elements / $\mathrm{mm}^{3}, 80 \%$ lymphocytes, $13 \%$ polymorphonuclear and 7\% macrophages, low glycosylation, low chlorouracil. A pulmonary X-ray reveals intense bilateral perihilar interstitial infiltration. A CT examination of the skull and brain revealed no changes.

Following epidemiological data (the patient originates from an area with above average incidence of pulmonary TB), clinical changes in CSF and pulmonary lesions, suspicion of tuberculous meningitis arises and antibiotic, antitubercular, corticoid, symptomatic and re-balancing infusions are initiated. Repeating the lumbar puncture, CSF was opalescent, with 436 elements, $10 \%$ neutrophils, $87 \%$ lymphocytes, macrophages 3\%, and low clorurorahia and glycorrhachia, as well as non-detectable Mycobacterium tuberculosis DNA.

In evolution, the patient's condition remains influenced, with persistent fever and headache, refuses nutrition, abdominal pain persist. The clinical examination revealed congestive pharyngitis and tonsillar hypertrophy grade 2 (beta-hemolytic Streptococcus absent). Neurosurgical examination is required and based on clinical data and CT craniocerebral interpretation an emergency intervention is excluded at that time. A third lumbar puncture is performed, this time under Midazolam anesthesia, revealing clear CSF with 840 elements / mm3, 75\% PMN, $22 \%$ lymphocytes, 3\% macrophages, low glucorrhagia and chlorochloride, and undetectable Mycobacterium tuberculosis DNA.

Other laboratory investigations reveal inflammatory biological syndrome, normocytic anemia, thrombocytosis, absent hemocultures after 7 days of incubation. A lingual rash revealed the presence of Candida albicans, and pulmonary radiographs revealed a bilateral hyaline adenopathy, <15mm diameter, as well as micronodular opacities with low intensity positioned in the right subclavicular area and in the right upper left lobe.

After 6 weeks of tuberculostatic therapy and cerebral anti-edematous treatment, the patient has a good overall condition, with no neurological and neuropsychic phenomena, without fever (kept febrile for 4 weeks). It is recommended to perform a control lumber puncture which showed clear CSF, 94 elements / mmc, 65\% lymphocytes, normal chemistry and Mycobacterium tuberculosis DNA undetectable.

TB ambulatory is being contacted, deciding discharge of the patient and continued chemoprophylaxis treatment at home for six months. Discharge recommendations are to avoid prolonged exposure to sunlight, TV, computer, avoiding consumption of neuroexcitants and control every month.

The following three evaluations were performed in the hospital ambulatory. At one month, the patient maintains a relatively good general condition, without fever, without subjective accusations and paraclinical analyzes are in normal limits, with the gradual resorption of pulmonary opacity revealed radiologically. Because of the affection suffered, the patient has a state of irritability and attention and concentration disorders, which required repeated sessions of psychotherapy with parents.

Because TBM symptoms can often be like those of flu, their persistence should raise some cause for concern. Fever, vomiting, lethargy, low appetite and weight loss may be the onset of tuberculous meningitis.

Even if chest x-ray is normal or quasi-normal at first, it does not necessarily mean that we do not have a tuberculosis case, as was the case with our patient. A lower concentration of glucose in the cerebrospinal fluid distinguishes TBM from viral meningitis, which is less dangerous. Research by scientists has also shown that measuring blood glucose at the same time can improve diagnosis (4).

If not treated, the decline can be sudden and shocking.

Early diagnosis and treatment can dramatically reduce the increased mortality associated with this disease. Etiotropic treatment of tuberculous meningitis begins not in diagnostic assertions, but in the suspicion suggested by clinical manifestations and cerebrospinal fluid (CSF) cytobiochimic changes. Until the bacteriological confirmation can be obtained, the absence of therapy causes the death to occur within three weeks. Generally, treatment should last for at least nine months and contain tuberculostatics in the quadruple combination. Adjuvant treatment with corticosteroids should be considered (9).

\section{Conclusions}

In the case we presented, the clinical data and the epidemiological context of tuberculosis in the area from which the patient originated could have guided the diagnosis. The certainty of a diagnosis provided by PCR was not possible due to the small amount of genetic material at the CSF level.

The only data that was decisive for establishing the diagnosis and evolution of the patientunder tuberculostatic therapy were the biochemical data, the CSF, which were strongly modified (glycorychia, chlorurocharya, albuminorahia).

In practice, the bacteriological diagnosis is not helpful, because of a minimum of 21 days for the growth of bacteria on the Lowenstein-J ensen culture medium, and the CSF $P C R$ is a sensitive method, but reserved only to the laboratories in the university or reference centers. However, in the CRL, there must be a minimum threshold of genetic material and the cost of the method is very high.

The patient whose case we presented survived due to the rapid establishment of the therapy, because the suspicion of BK etiology was based on only modified biochemical data. It is essential to recognize this pathology from the first 10 days of onset, because overcoming this term leads to mortality in most of the cases.

Particularity of the case: relatively sudden onset with rapid evolution of neurological and psychological impairment in a child without a history of heredocolateral tuberculosis but which originates from an endemic area for this pathology. Major residual behavioral disorders required repeated psychotherapy sessions. 
Prognosis is a good one, considering family cooperation, good compliance with treatment, and full remittance of neurological deficits.

\section{References}

1.** http://apps.w ho.int/iris/bitstream/10665/259366/1/9789241565516eng.pdf?ua $=1$

2.*** http://www.who.int/tb/publications/global_report/en

3.J AIN, S. K., ORDONEZ, A., KINIKAR, A., et al. (2013). Pediatric tuberculosis in young children in India: a prospective study. BioMed research international, 2013.

4.J ENNIFER J UBULIS, SANJAY K. JAIN (2016). Tuberculous meningitis. The Clinical Advisor

5.MELINTE, V., SIMION, V., HOMOS, etal. (2007). Meningita tuberculoasã oi cascada complicabilor. Romanian Journal of Infectious Diseases, 10(4).
6.MIFTODE EG, DORNEANU OS, LECA DA, et al. (2015) Tuberculous Meningitis in Children and Adults: A 10-Year Retrospective Comparative Analysis. PLOS ONE 10(7)

7.TOROK, M. E. (2015). Tuberculous meningitis: advances in diagnosis and treatment. British medical bulletin, 113(1), 117-131.

8.LUCA MC, VATA A, MIFTODE E. Diagnosticul precoce al meningitei tuberculoase. Infection.ro, 2008, 2: 28-32.

9.ISRANI AV, DAVE DA, MANDAL $A$, et al. Tubercular meningitis in children: Clinical, pathological, and radiological profile and factors associated with mortality. J ournal of Neurosciences in Rural Practice. 2016;7(3):400-404

10.Van BUI T, LEVY-BRUHL D, CHE D, et al. (2015)Impact of the BCG vaccination policy on tuberculous meningitis in children under 6 years in metropolitan France between 2000 and 2011. Euro Surveill 20(11)

11.*** WHO Report 2013. Global tuberculosis control. Geneva, WHO, 2013: 1-289.

Manuscript received: 27.11 .2019 